\title{
Blood Biomarkers Dynamic Detection, Especially Eosinophils has Certain Value in the Clinical Diagnosis, Severity Classification and Prognosis Judgment of COVID-19
}

Quan Zhou

suizhou central hospital

Qiang Huang

Suizhou central hospital https://orcid.org/0000-0003-0630-6353

Long Nie

Suixian people's hospital

Pin Ren

suixian peolple's hospital

\section{Yue Xu}

Suizhou central hospital

Li Xiao

suixian people's hospital

\section{Yanlong Yang}

suixian peolple's hospital

Bo Chen ( $\nabla$ wercan@sina.com.cn )

suizhou central hospital

Mingli Tu ( $\nabla 13337191 @ q q . c o m$ )

suizhou central hospital

Research

Keywords: leucocytes, lymphocyte, eosinophils, COVID-19

Posted Date: June 12th, 2020

DOl: https://doi.org/10.21203/rs.3.rs-34268/v1

License: (1) (1) This work is licensed under a Creative Commons Attribution 4.0 International License. Read Full License 


\section{Abstract}

In December 2019, coronavirus disease 2019 (COVID-19) was first found in Wuhan, China and soon was reported all around the world. Novel coronavirus (COVID-19) is highly infectious and requires early detection, isolation, and treatment. We tried to find some useful information by analyzing the covid-19 screening data, so as to provide help for clinical practice. In this prospective study, we retrospectively analyzed the clinical data of 131 patients with COVID-19 and 119 controls. For confirmed cases, the data of blood routine examination were analyzed among severe patients and non-severe group. The blood routine examination results were dynamically observed in the survivors and nonsurvivors. We find that patients with COVID-19 have lower counts of leucocytes, lymphocytes, eosinophils, which were compared with controls $(P<0.001)$. In severe group, patients have the lower count of lymphocytes and eosinophils, but the higher leucocytes count (all $P$ values $<0.01$ ). Eosinophils have high diagnostic efficacy analysis of severe COVID-19, and its area under the curve reached 0.750 . Patients whose eosinophils returned to normal early had significantly longer survival times than those who $\operatorname{did} \operatorname{not}(P<0.001)$. Patients with COVID-19 have abnormal peripheral blood routine examination results. Dynamic surveillance of peripheral blood system especially eosinophils is helpful in the diagnosis, assess the prognosis and prediction of severe COVID-19 cases.

\section{Background}

The new coronavirus pneumonia (COVID-19) is highly contagious and has spread worldwide[1]. Some patients deteriorated rapidly and developed acute respiratory distress syndrome (ARDS), and even died. Therefore, improving the treatment level of COVID-19 has become a major challenge for clinicians, and it is more important to explore convenient and effective clinical monitoring indicators[2]. The release of the first to seventh editions of China's new coronary pneumonia diagnosis and treatment program has played an extremely important role in the control of China's COVID-19 epidemic. Each version of the guideline regards "the total number of peripheral white blood cells in the early stage of the disease is normal or decreased, and the lymphocyte count is normal or decreased" as one of the diagnostic basis[3]. In clinical practice, we found that the proportion of Eosinophils (EOS) in COVID-19 patients decreased at an early stage, and recovered with the recovery of the disease, and the patients who died rarely recovered before death. In order to explore the value of EOS dynamic detection in the clinical diagnosis, classification and prognosis of new coronavirus pneumonia (COVID-19), we retrospectively analyzed the clinical data of 131 patients with COVID-19.

\section{Materials And Methods}

\section{Patients' involvement and data collection}

This case series was approved by the institutional ethics board of Suizhou Central Hospital. All consecutive patients with confirmed NCIP admitted to Suizhou Central Hospital from February 1 to March 15, 2020, were enrolled. Oral consent was obtained from patients. Suizhou Central Hospital, located in 
Suizhou, Hubei Province, the endemic areas of NCIP, is one of the major tertiary teaching hospitals and is responsible for the treatments for NCIP assigned by the government. All patients with NCIP enrolled in this study were diagnosed according to Diagnosis and treatment of novel coronavirus infected pneumonia (trial 7th edition)[3].

131 cases of COVID-19 were diagnosed as case group. According to the latest new coronary diagnosis and treatment plan, they were divided into nonsevere group and severe group. Mild and common types were included in nonsevere group, and severe and critically severe were included in severe group. Among them, 94 cases were in the nonsevere group (all patients were cured and discharged), 37 were in the severe group (all patients died after treatment), 67 were male and 64 were female. The average age is 51 \pm 17 years old. In the non-COVID-19 group, there were 50 males and 69 females, with an average age of $42 \pm 16$ years. And we collected the laboratory test data from the hospital information system for 250 patients in the two groups.

\section{Real-Time Reverse Transcription Polymerase Chain Reaction Assay for COVID-19}

Throat swab samples were collected for extracting COVID-19 RNA from patients suspected of having COVID-19 infection. After collection, the throat swabs were placed into a collection tube with $150 \mu \mathrm{L}$ of virus preservation solution, and total RNA was extracted within 2 hours using the respiratory sample RNA isolation kit. In brief, $40 \mu \mathrm{L}$ of cell lysates were transferred into a collection tube followed by vortexfor 10 seconds. After standing at room temperature for 10 minutes, the collection tube was centrifugated at $1000 \mathrm{rpm} / \mathrm{min}$ for 5 minutes. The suspension was used for real-time reverse transcription polymerase chain reaction (RT-PCR) assay of 2019-nCoV RNA. Two target genes, including open reading frame $1 \mathrm{ab}(\mathrm{ORF} 1 \mathrm{ab})$ and nucleocapsid protein(N),were simultaneously amplified and tested during the real-time RT-PCR assay. Target 1(ORF1ab): forward primer CCCTGTGGGTTTTACACTTAA; reverse primer ACGATTGTGCATCAGCTGA; and the probe5'-VIC-CCGTCTGCGGTATGTGGAAAGGTTATGG-BHQ1-3'. Target 2 (N): forward primer GGGGAACTTCTCCTGCTAGAAT; reverse primer CAGACATTTTGCTCTCAAGCTG; and the probe5'-FAM- TTGCTGCTGCTTGACAGATT-TAMRA-3'. The real-time RT-PCR assay was performed using a 2019-nCoV nucleic acid detection kit according to the manufacturer's protocol(Nanjing Vazyme Biotech Co., Ltd). Reaction mixture contains $18 \mu \mathrm{L}$ of reaction buffer, $2 \mu \mathrm{L}$ of enzyme solution and $5 \mu \mathrm{L}$ of negative control (use without extraction), positive control (use without extraction) or sample nucleic acid extract. RT-PCR assay was performed under the following conditions: incubation at $50^{\circ} \mathrm{C}$ for 15 minutes and $95^{\circ} \mathrm{C}$ for 30 seconds, 45 cycles of denaturation at $94^{\circ} \mathrm{C}$ for 10 seconds, and extending and collecting fluorescence signal at $58{ }^{\circ} \mathrm{C}$ for 30 seconds. A cycle threshold value(Ct-value) less than 38 was defined as a positive test result, and a Ct-value of 40 or more was defined as a negative test. A medium load, defined as a Ct-value of 38 to less than 40 , required confirmation by retesting.

\section{Statistical Analysis}


The median and inter quartile range were calculated for continuous variables, and the two-tailed t-test and Mann-Whitney test were used as appropriate to compare continuous variables in data from different patient groups. The frequencies of categorical variables were compared using the chi-square and Fisher's exact test as appropriate. $P$ values less than 0.05 were considered to indicate statistically significant differences. SPSS version24.0 (SPSS, Inc., Chicago, IL, USA) was used for data analysis. Statistical analysis graphs were generated and drawn using GraphPad Prism 8.00 software (GraphPad, Inc., La Jolla, CA, USA). ROC curve analysis was generated and drawn using MedCalc 19.2 statistical software (MedCalc,Inc., Ostend,Belgium ).

\section{Results}

\section{Basic demographic factors and blood biomarkers in the COVID-19 and nonCOVID-19 groups}

The median age (range) was 50.56 years (25-82 years) in patients with COVID-19 and 41.87 years (17-85 years) in the controls. And there was no significant difference in the sex ratio between the two groups. As for laboratory findings, COVID-19 patients had significantly lower levels of leukocytes $\left(5.18 \times 10^{9} / \mathrm{L}\right)$, lymphocytes $\left(1.08 \times 10^{9} / \mathrm{L}\right)$, eosinophils $\left(0.01 \times 10^{9} / \mathrm{L}\right)$ compared with the controls. When dichotomized into binary variables, eosinopenia $\left(<0.02 \times 10^{9} / \mathrm{L}\right)$ and lymphocyte $\left(<1.1 \times 10^{9} / \mathrm{L}\right)$ decline were present in $57.2 \%$ and $55.7 \%$ of COVID-19 patients, much higher than $35.2 \%$ and $43.7 \%$ of the controls, respectively. Meanwhile, $92.3 \%$ of the patients with COVID-19 and $84.0 \%$ of the controls had normal or decreased number of leukocytes and/or lymphopenia, two recommended parameters in the guideline.(As shown in Table 1) 


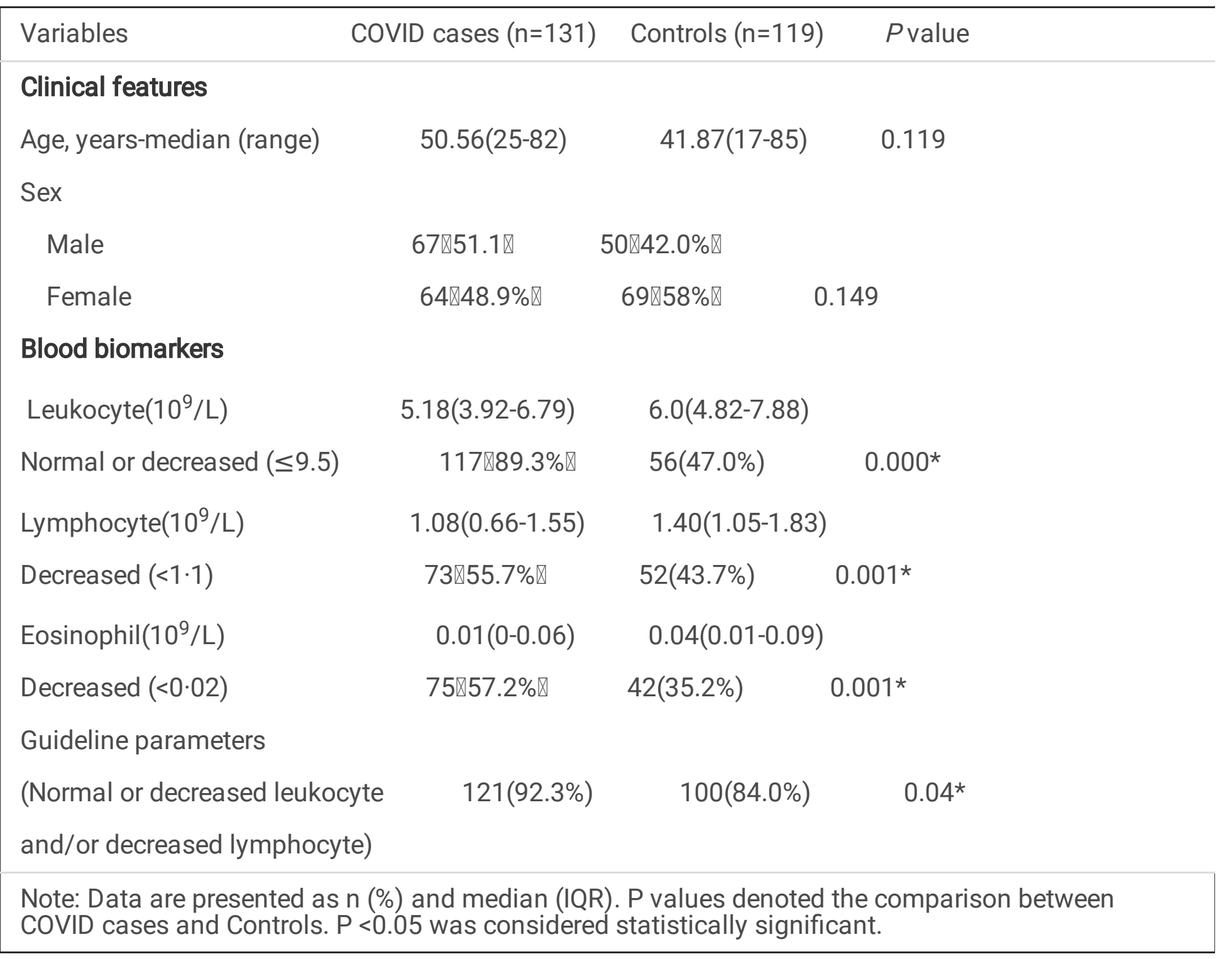

Table 1

Comparison of clinical features and blood biomarkers between patients with and without COVID-19

We further evaluated the predictive capacity of those significant blood biomarkers (leukocytes, lymphocytes and eosinophils). Among the 3 biomarkers, we found that the eosinopenia produced the highest specificity $(90.4 \%)$, PPV $(88.3 \%)$, and AUC (0.825). We found that the combination of eosinopenia and lymphocyte decline yielded the highest specificity (91.6\%), PPV (89.5\%), and AUC (0.843).(As shown in Table 2 and Figure 1) 


\begin{tabular}{|c|c|c|c|c|c|c|c|}
\hline $\begin{array}{l}\text { Prediction } \\
\text { models }\end{array}$ & AUC & SE & $95 \% \mathrm{Cl}$ & Sensitivity & Specificity & PPV & NPV \\
\hline Leu $(\leq 9.5)$ & 0.696 & 0.034 & $\begin{array}{l}0.635- \\
0.752\end{array}$ & $56.5 \%$ & $85.7 \%$ & $81.3 \%$ & $64.2 \%$ \\
\hline $\operatorname{Lym}(<1.1)$ & 0.794 & 0.027 & $\begin{array}{l}0.739- \\
0.843\end{array}$ & $68.7 \%$ & $75.6 \%$ & $75.6 \%$ & $68.7 \%$ \\
\hline Eos $(<0.02)$ & 0.825 & 0.026 & $\begin{array}{l}0.772- \\
0.870\end{array}$ & $63.4 \%$ & $90.4 \%$ & $88.3 \%$ & $69.7 \%$ \\
\hline $\begin{array}{l}\text { Eos }(<0.02) \text { and } \\
\text { Lym }(<1.1)\end{array}$ & 0.843 & 0.024 & $\begin{array}{l}0.792- \\
0.886\end{array}$ & $64.9 \%$ & $91.6 \%$ & $89.5 \%$ & $70.3 \%$ \\
\hline
\end{tabular}

Table 2

Diagnostic performance of single and combined blood biomarkers on differentiating patients with COVID$19(n=131)$ from controls patients $(n=119)$

\section{Basic demographic factors and blood biomarkers in the nonsevere and severe groups}

The median age (range) was 43.6 years (27-72 years) in the nonsevere patients and 68 years (36-82 years) in the severe patients with COVID-19. We found patients in the severe group are significantly older than those in the nonsevere group. And there was still no significant difference in the sex ratio between the two groups. As for laboratory findings, severe patients had significantly higher levels of leukocytes $\left(7.4 \times 10^{9} / \mathrm{L}\right)$ than nonsevere group and had lower levels of lymphocytes $\left(0.45 \times 10^{9} / \mathrm{L}\right)$, eosinophils $\left(0.00 \times 10^{9} / \mathrm{L}\right)$ compared with the nonsevere patients. When dichotomized into binary variables, lymphocyte $\left(<1.1 \times 10^{9} / \mathrm{L}\right)$ and eosinopenia $\left(<0.02 \times 10^{9} / \mathrm{L}\right)$ count decline were present in $83.7 \%$ and $91.8 \%$ of severe patients, much significantly higher than $37.2 \%$ and $43.6 \%$ of the nonsevere patients, respectively. (As shown in Table 3) 


\begin{tabular}{|c|c|c|c|}
\hline Variables & Nonsevere patients & Severe patient & $P$ value \\
\hline \multicolumn{4}{|l|}{ Clinical features } \\
\hline Age, years-median (range) & $43.6(27-72)$ & $68(36-82)$ & $0.000^{\star}$ \\
\hline$>50$ year & $35(37.2 \%)$ & $34(91.8 \%)$ & $0.000^{*}$ \\
\hline \multicolumn{4}{|l|}{ Sex } \\
\hline Male & $49 \llbracket 52.1 \% \rrbracket$ & $18 \rrbracket 48.6 \% \rrbracket$ & \\
\hline Female & $45 \rrbracket 47.9 \% \rrbracket$ & $19 \rrbracket 51.4 \% \rrbracket$ & 0.720 \\
\hline \multicolumn{4}{|l|}{ Blood biomarkers } \\
\hline Leukocyte $\left(10^{9} / \mathrm{L}\right)$ & $4.76(3.63-5.93)$ & \multicolumn{2}{|c|}{$7.40(5.15-10.5)$} \\
\hline Normal or decreased $(\leq 9.5)$ & $90 \rrbracket 95.7 \% \rrbracket$ & $27(72.9 \%)$ & $0.000^{\star}$ \\
\hline Lymphocyte(10\%/L) & $1.30(0.92-1.67)$ & \multicolumn{2}{|c|}{$0.45(0.29-0.69)$} \\
\hline Decreased $(<1 \cdot 1)$ & $35 \llbracket 37.2 \% \rrbracket$ & $31(83.7 \%)$ & $0.000 *$ \\
\hline Eosinophil $\left(10^{9} / \mathrm{L}\right)$ & $0.03(0.01-0.09)$ & \multicolumn{2}{|c|}{$0.00(0.00-0.01)$} \\
\hline Decreased $(<0.02)$ & $41(43.6 \%)$ & $34(91.8 \%)$ & $0.000^{*}$ \\
\hline
\end{tabular}

\section{Table 3}

Comparison of clinical features and blood biomarkers between nonsevere and severe patients with COVID-19

We further evaluated the predictive capacity of eosinophils differentiating nonsevere and severe patients with COVID-19. We found that the result of specificity (86.2\%), PPV (63.9\%) and AUC (0.750). (As shown in Table 4 and Figure 2)

\begin{tabular}{|llllllll}
\hline Prediction models & AUC & SE & $95 \% \mathrm{Cl}$ & Sensitivity & Specificity & PPV & NPV \\
\hline EOS $(<0 \cdot 02)$ & 0.750 & 0.049 & $0.667-0.822$ & $62.2 \%$ & $86.2 \%$ & $63.9 \%$ & $85.3 \%$
\end{tabular}

The unit is $10^{9} / \mathrm{L}$ for Eos. AUC, area under the curve; COVID-19, coronavirus disease; Eos: eosinopenia; NPV, negative predictive value; PPV, positive predictive value

Table 4

Diagnostic performance of Eosinophil on differentiating nonsevere and severe patients with COVID-19 


\section{Blood biomarkers dynamic changes of patients with COVID- 19}

We randomly selected 20 survivors and 20 nonsurvivors from the patients with COVID-19, and analyzed their dynamic changes in blood biomarkers over time. As shown in Figure 3A, although the white blood cell count of the survivors increased temporarily in the early stage, it gradually decreased to the normal level later. The level of white blood cell count of the nonsurvivors increased in the early stage, although it decreased afterwards, but eventually gradually increased to a higher level. In Figure 3B, we found that the lymphocyte count of survivors showed a gradual upward trend with time. In the figure we found that around the 17th day, the cell count rose to normal levels. However, the lymphocyte count of the nonsurvivors has been at a low level, and the increase is not obvious. From Figure $3 \mathrm{C}$, we can see that the eosinophil count of the survivors rose quickly, rose to normal levels around the fifth day, and then continued to increase. However, the eosinophil count of the nonsurvivors continues to be low, below normal levels.

\section{Relationship between blood biomarkers and recovery of patients with COVID-19}

In the cured group, there were 75 patients with eosinophil count and 73 patients with lymphocyte count below the lower limit of normal before treatment. In these patients, the average time for eosinophil count to return to normal was 6.2(4.6-7.8) days, the average time for lymphocytes to return to normal levels was 18.5(16.7-20.6) days, and the average time for nucleic acid to turn negative was 17.2(15.5-19.0) days. Data are presented as median (IQR).

In order to study the relationship between blood biomarkers and recovery of COVID-19 patients, we selected two surviving cases. As shown in Figures $4 A$ and $B$, these are the results of chest $C T$ imaging taken at different times after the patient was admitted to our hospital. Case 1 is a 34-year-old young man and case 2 is a 57-year-old middle-aged male. Figures $4 \mathrm{C}$ and $\mathrm{D}$ are the dynamic changes of blood biomarkers in case 1 and case2, respectively.

In case 1, the patient was transferred from the external hospital and the chest CT was examined in the external hospital, so there was no imaging examination on the first day of admission. We found that on the 14th day, from the lung $C T$, the patient's lungs were still very heavy, but the white blood cells had dropped to normal, eosinophils had risen to normal, but the lymphocytes were still below normal. On the 25th and 27th day of admission, the patient's pharyngeal swab was negative for nucleic acid inspection, but the patient's lung CT showed a slight exudative lesion. At this time, the lymphocytes did not increase to the normal range. The lymphocyte count did not reach normal levels until the lung CT showed that the exudative lesions were significantly improved. 
In case 2, the patient's lung CT showed exudative lesions after admission, and the lung infection continued to increase until day 26 . But through active treatment, the patient's lymphocyte and eosinophil counts continue to rise, and eosinophils have risen to normal levels on about day 7.On day 35 and 37, the patient's throat swab nucleic acid test showed negative results. At this time, the patient's white blood cells have dropped from the previous high level, and the lymphocyte count has gradually increased to normal.

\section{Relationship between recovery of eosinophil count and patient prognosis}

Finally, according to the recovery of eosinophils, we divided these patients with COVID-19 into two groups. One group $(n=14)$ is a group with eosinophils continuously lower than normal level, and the other group $(n=13)$ is a group with eosinophils level elevated to normal. We counted the one-month survival of these two group patients and made survival curves, as shown in Figure 5 . We found that patients whose eosinophils level has not recovered have significantly shorter survival times than those who recovered.

\section{Discussion}

COVID-19 is a systemic multiple organ injury disease caused by severe acute respiratory syndrome coronavirus 2 (SARS-CoV-2), with the lung as the main target organ. While most people with COVID-19 develop only mild or uncomplicated illness, approximately $14 \%$ develop severe disease that requires hospitalization and oxygen support, and $5 \%$ require admission to an intensive care unit[4]. In severe cases, it can cause severe lung injury and acute respiratory distress syndrome (ARDS), and even death[5]. In the seventh version of the new coronavirus diagnosis and treatment plan, it was pointed out that the total number of peripheral blood leukocytes in patients at the early stage of the disease was normal or decreased, and the lymphocyte count decreased; in severe cases, the peripheral blood lymph count decreased progressively[3]. Lymphopenia is a common feature in the patients with COVID-19 and might be a critical factor associated with disease severity and mortality[6]. In the pathology study of COVID-19 patients, Zhe Xu et al. reported that the counts of peripheral CD4 and CD8 T cells were substantially reduced [7].COVID-19 will cause abnormalities of the parameters of peripheral blood routine. The decrease in lymphocytes is the most obvious abnormalities, which is related to poor outcome of COVID$19[8]$. In our study, we analyzed the data of blood routine examination of cases with COVID-19 and found that the lower count and delay in increasing of eosinophils can also be relevant to the severity of the disease and clinical classification.

Eosinophils normally account for only a small percentage of circulating leukocytes (1-3\%), but their levels can vary in a variety of disease states[9]. Their level is clinically relevant as eosinophils are potent proinflammatory cells and they have pleotropic roles as regulatory cells involved in protective immunity[10], including antiviral responses and shaping diverse physiological responses[11]. Interestingly, Zhang et al. reported eosinopenia (52.9\%) were observed in most patients. Blood eosinophil counts correlate positively with lymphocyte counts in severe and nonsevere patients after hospital 
admission[12]. Similarly, Qian et al. reviewed the 91 medical records of cases of COVID-19 and noted that 47(51.65\%) had decreased eosinophils count[13]. Our study found that in COVID-19 patients of Suizhou area, the total number of lymphocytes and eosinophils in the initial stage of the disease both decreased, and the proportion of people with decreased eosinophil counts was slightly higher. At the same time, we also made ROC curves. In the diagnosis of COVID-19, the AUC and specificity of eosinophils are higher than that of lymphocytes and leukocytes. When eosinophils are combined with lymphocytes, the diagnosis effect has higher AUC and specificity. This result is consistent with previous research[14], which demonstrated that eosinophils count is helpful to speed up diagnosis for COVID-19 patients, especially in combination with lymphocyte count.

We have also carried out relevant research in severe patients and non-severe patients in COVID-19 patients. In critically ill patients, lymphocyte counts and eosinophil counts were significantly lower, with $83.7 \%$ of patients having decreased lymphocyte counts and $91.8 \%$ of patients having decreased eosinophil counts. Most of the critically ill patients have eosinophil counts as low as 0 . However, the white blood cell count of critically ill patients is higher than that of non-critically ill patients. Qin et al. reported similar findings, indicating the potential critical condition and serious disturbance of internal environment in those severe infected cases[15]. Li et al. found that some death cases of COVID-19 have superimposed bacterial pneumonia[16]. Bacterial infection leads to significant increased leucocyte count and neutrophil count. So we speculate that the increase in the white blood cell count in critically ill patients may be caused by the later combined bacterial infection. This needs further research to confirm. We further prepared an ROC curve for eosinophils to distinguish between severe and non-severe patients, with higher specificity, PPV and AUC. According to the above research, we speculated that the eosinophils count has certain value in judging the severity of COVID-19.

In order to understand the role of various parameters of peripheral blood cells in patient's prognosis, our study tracked and analyzed these parameters on admission, day 1, day 3, day 13, day 15 and day 20 in the survivor and non-survivor group, and drew a curve with the median value. Our research found that the survivor's white blood cell count gradually became normal after treatment. However, the white blood cell count of the deceased gradually increased, which is the same as the previous research results of severe patients. It may be related to cytokine storm induced by virus invasion or complicated by later bacterial infection, so the prognosis is extremely poor. The specific reason remains unclear. As for lymphocytes and eosinophils, the blood test results of the survivors have increased almost every day throughout the treatment. And among the dead, they continue to be at a low level. This result is consistent with previous research[8]. These abnormalities suggest that 2019-nCoV infection may be associated with cellular immune deficiency, which is similar to those previously observed in patients with MERS-CoV and SARSCoV infection[17]. In our study, we also found that among survivors, lymphocytes reached normal values around day 17, while eosinophils reached normal levels around day 5. This shows that the eosinophils of the COVID-19 patients rose to normal levels in the early stage, indicating a good prognosis. Therefore, the total number of eosinophils is an important indicator of the patient's prognosis, while the predictive effect of lymphocyte count has a certain lag. 
In order to better clarify the relationship between blood biomarkers and patient prognosis, we selected 2 cases with a better prognosis. From these two cases, we found that when the nucleic acid result turned negative, the acute exudation of the lungs of the two people had been significantly absorbed in CT scan, and the white blood cell count of both people had dropped to normal levels. After the nucleic acid test turned negative, the patient's lymphocyte count returned to normal levels; while eosinophils had risen to normal some time before the throat swab nucleic acid test turned negative. From case 2, we also found that when eosinophils rose to normal levels, the lung exudation was still worse. Only when the lymphocytes gradually increase, the lung condition slowly improves. Therefore, the increase of eosinophil count has a certain value for the evaluation of the patient's prognosis, but it is better to be combined with other factors such as lymphocyte count, CD8+ T cells[18], high SOFA score, and D-dimer[19].

Finally, we made a survival curve based on the recovery of patients' eosinophils. From the results, we can see that the prognosis of patients with eosinophil count recovery is much better. However, persistent low eosinophil could help clinicians to identify patients with poor prognosis at an early stage.

There were several limitations to our study that might cause some potential bias. First, it was a retrospective, single-center, small-sample study of patients admitted to the hospital; standardized data for a larger cohort would be better to assess the temporal change in immune response after infection with COVID-19. Second, Many COVID-19 patients are elderly patients with various chronic diseases in the past. The patient's different past medical history has a certain influence on the comparative analysis of blood routine.

\section{Conclusion}

Despite that, our study demonstrated several novel findings on the relationship between blood biomarkers and patients with COVID-19. Especially eosinophils, it is helpful in the early screening of critical illness and diagnosis and assessing the prognosis of COVID-19.

\section{Declaration}

\section{Ethics approval and consent to participate}

Prior to its start, the study protocol was approved by the ethics committee of Suizhou Central Hospital, Hubei University of Medicine, Suizhou, China. Verbal informed consent about the study procedures were obtained from all the subjects before the trial.

\section{Consent for publication}

Not applicable 


\section{Availability of data and materials}

The datasets, as well as the patent materials, used and analyzed during the current study are available from the corresponding author on reasonable request.

\section{Funding}

This research did not receive any specific grant from funding agencies in the public, commercial, or notfor-profit sectors.

\section{Competing interests}

The authors declare that they have no competing interests.

\section{Authors' contributions}

Mingli Tu is the guarantor of this research. Bo Chen and Mingli Tu were responsible for study concept and design. Yanlong Yang and Long Nie contributed to patient recruitment and patient follow-up. Li Xiao was responsible for outcome measurement. Pin Ren and Yue Xu finished data acquisition. Quan Zhou and Qiang Huang finished data analysis. All authors contributed to the drafting of this manuscript. All authors read and approved the final manuscript.

\section{Acknowledgements}

We acknowledge all health-care workers involved in the diagnosis and treatment of patients in Suizhou. Special thanks to Director Xu and Director Wang for giving us some guidance in the research process.

\section{References}

1. Singhal T: A Review of Coronavirus Disease-2019 (COVID-19). Indian J Pediatr 2020, 87:281-286.

2. Yun H, Sun Z, Wu J, Tang A, Hu M, Xiang Z: Laboratory data analysis of novel coronavirus (COVID19) screening in 2510 patients. Clin Chim Acta 2020, 507:94-97.

3. National Health Commission of the People's Republic of China. Diagnosis and treatment of novel coronavirus infected pneumonia (trial 7th edition) [EB/OL]. (2020-03-04) http://www.nhc.gov.cn/xcs/zhengcwj/202003/46c9294a7dfe4cef80dc7f5912eb1989.shtml.

4. Clinical management of severe acute respiratory infection when COVID-19 is suspected $\square$ Interim guidance. 13 March 2020. 
5. Guan WJ, Ni ZY, Hu Y, Liang WH, Ou CQ, He JX, Liu L, Shan H, Lei CL, Hui DSC, et al: Clinical Characteristics of Coronavirus Disease 2019 in China. N Engl J Med 2020, 382:1708-1720.

6. Jasper Fuk-Woo Chan, Shuofeng Yuan, Kin-Hang Kok, Kelvin Kai-Wang To, Hin Chu et al:A familial cluster of pneumonia associated with the 2019 novel coronavirus indicating person-to-person transmission. Lancet 2020;395:514-23.

7. Zhe Xu, Lei Shi, Yijin Wang, Jiyuan Zhang, Lei Huang et al :Pathological findings of COVID-19 associated with acute. Lancet Respir 2020.

8. Sun S, Cai X, Wang H, He G, Lin Y, Lu B, Chen C, Pan Y, Hu X: Abnormalities of peripheral blood system in patients with COVID-19 in Wenzhou, China. Clin Chim Acta 2020, 507:174-180.

9. Burris D, Rosenberg CE, Schwartz JT, et al. Pediatric Hypereosinophilia Characteristics, Clinical Manifestations, and Diagnoses. J Allergy Clin Immunol Pract 2019;7(8):2750-2758.e2.

10. Lindsley AW, Schwartz JT, Rothenberg ME: Eosinophil responses during COVID-19 infections and coronavirus vaccination. J Allergy Clin Immunol 2020.

11. Percopo CM, Dyer KD, Ochkur SI, et al. Activated mouse eosinophils protect against lethal respiratory virus infection. Blood. 2014;123(5):743-752.

12. Zhang JJ, Dong X, Cao YY, Yuan YD, Yang YB, Yan YQ, Akdis CA, Gao YD: Clinical characteristics of 140 patients infected with SARS-CoV-2 in Wuhan, China. Allergy 2020.

13. Qian GQ, Yang NB, Ding F, et al. Epidemiologic and Clinical Characteristics of 91 Hospitalized Patients With COVID-19 in Zhejiang, China : A retrospective, multi-centre case series. QJM. 2020;hcaa089.

14. Li Q, Ding X, Xia G, Chen HG, Chen F, Geng Z, Xu L, Lei S, Pan A, Wang L, Wang Z: Eosinopenia and elevated C-reactive protein facilitate triage of COVID-19 patients in fever clinic: a retrospective casecontrol study. EClinicalMedicine 2020:100375.

15. Qin C, Zhou L, Hu Z, Zhang S, Yang S, Tao Y, Xie C, Ma K, Shang K, Wang W, Tian DS: Dysregulation of immune response in patients with COVID-19 in Wuhan, China. Clin Infect Dis 2020.

16. Li X, Wang L, Yan S, Yang F, Xiang L, Zhu J, Shen B, Gong Z: Clinical characteristics of 25 death cases with COVID-19: A retrospective review of medical records in a single medical center, Wuhan, China. Int J Infect Dis 2020, 94:128-132.

17. Wang D, Hu B, Hu C, Zhu F, Liu X, Zhang J, Wang B, Xiang H, Cheng Z, Xiong Y, et al: Clinical Characteristics of 138 Hospitalized Patients With 2019 Novel Coronavirus-Infected Pneumonia in Wuhan, China. JAMA 2020.

18. Lin A, He ZB, Zhang S, Zhang JG, Zhang X, Yan WH. Early Risk Factors for the Duration of SARS-CoV2 Viral Positivity in COVID-19 Patients. Clin Infect Dis. 2020;ciaa490.

19. Zhou F, Yu T, Du R, et al.Clinical Course and Risk Factors for Mortality of Adult Inpatients With COVID19 in Wuhan, China A Retrospective Cohort Study. Lancet. 2020 Mar 28;395(10229):1054-1062.

\section{Figures}




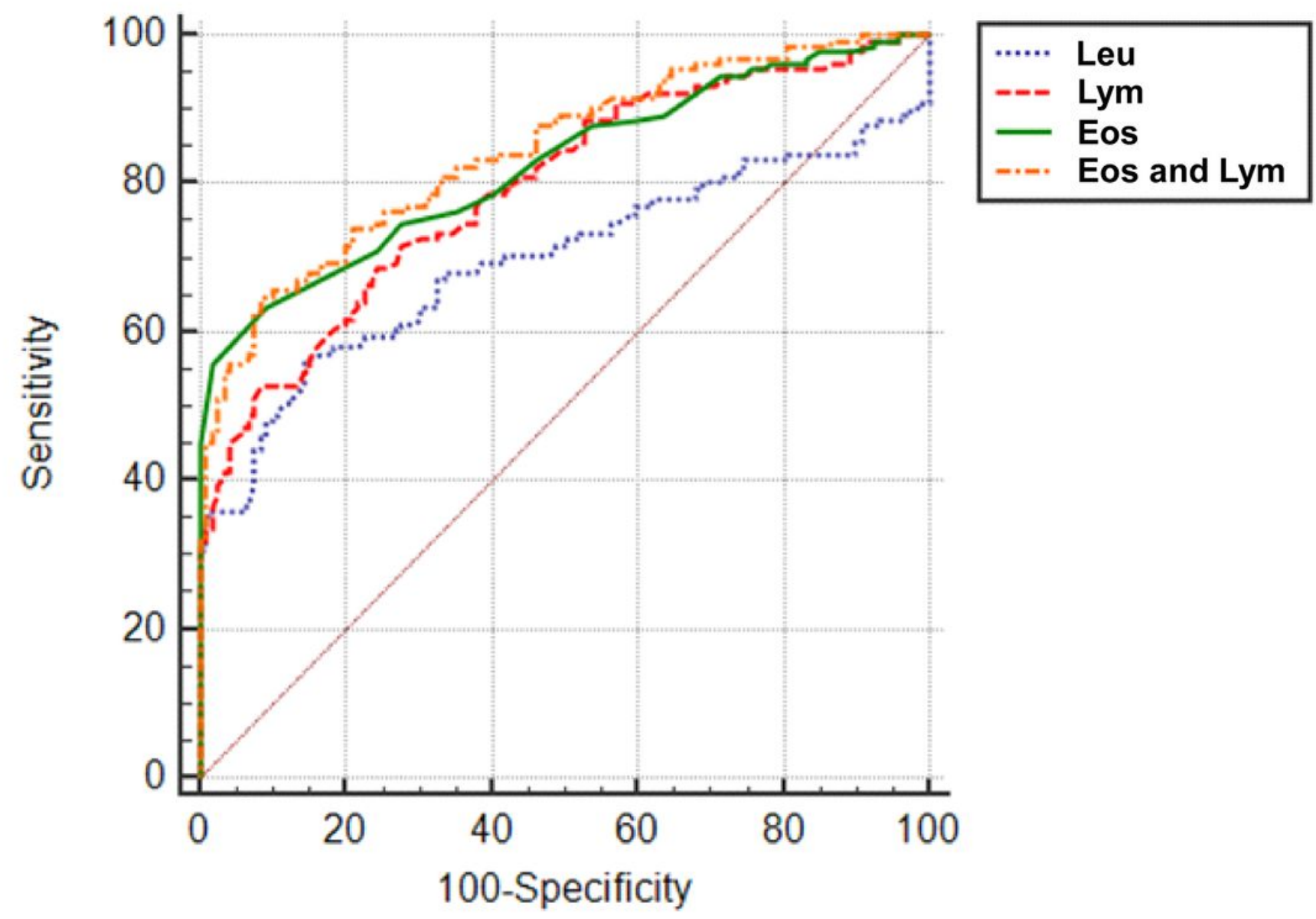

Figure 1

ROC curve of COVID-19 diagnosed by leukocyte, lymphocyte囚eosinophil and eosinophil combined with lymphocyte. Leu: leukocyte, Lym: lymphocyte, Eos: eosinophil. 


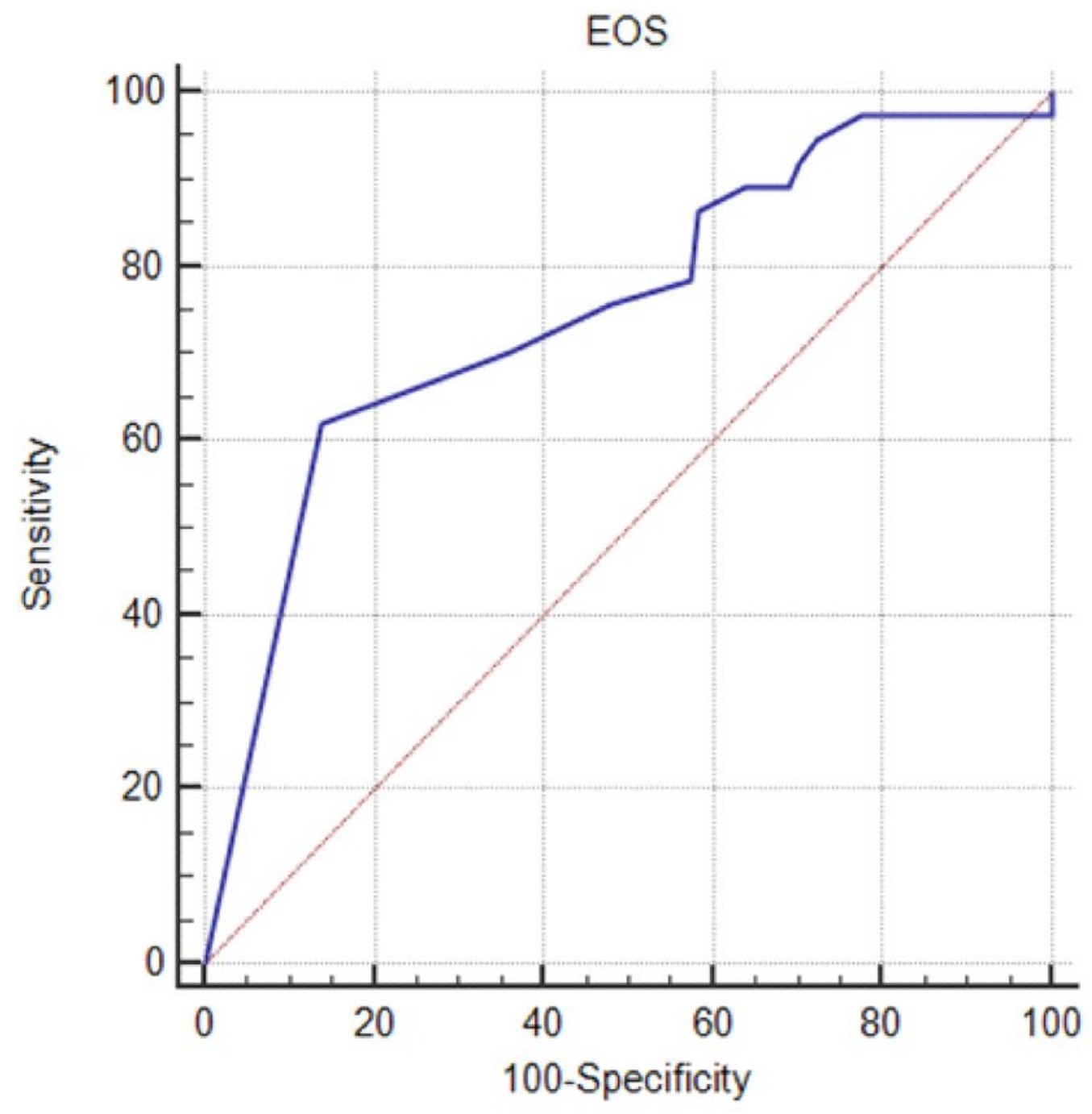

Figure 2

ROC curve of eosinophil differentiating severe and nonsevere types in COVID-19 patients.EOS: eosinophil.
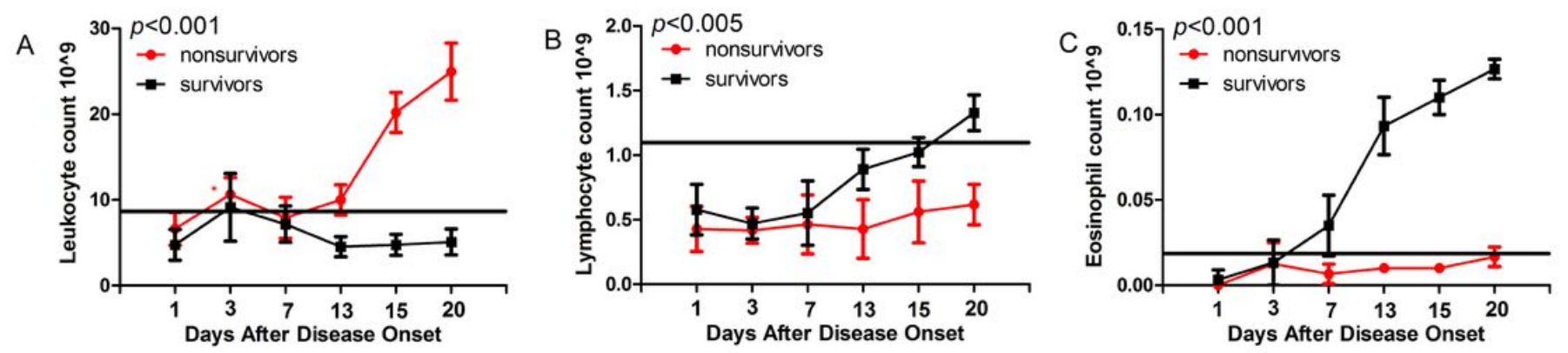

Figure 3 
Blood biomarkers dynamic changes of survivors $(n=20)$ and nonsurvivors $(n=20)$ with COVID-19 after disease onset. A. Leukocyte count change of survivors and nonsurvivors. B. Lymphocyte count change of survivors and nonsurvivors. C. Eosinophil count change of survivors and nonsurvivors.

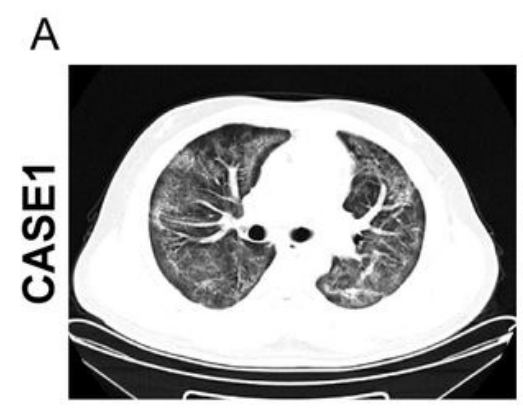

$\mathrm{B}$

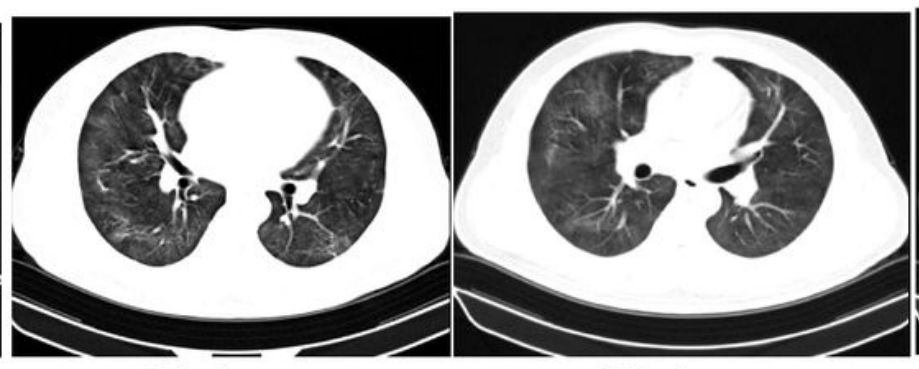

24 day
32 day

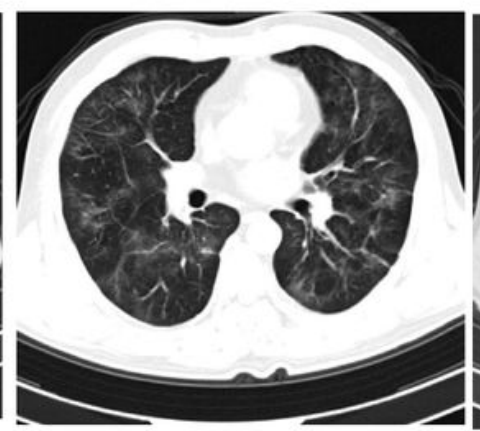

35 day

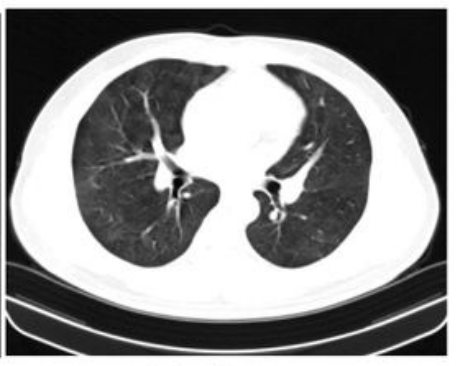

36 day

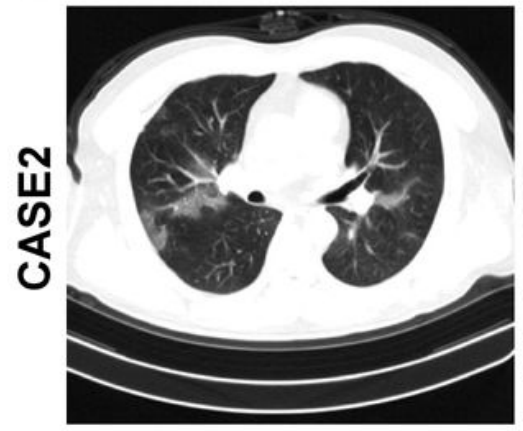

1 day

C

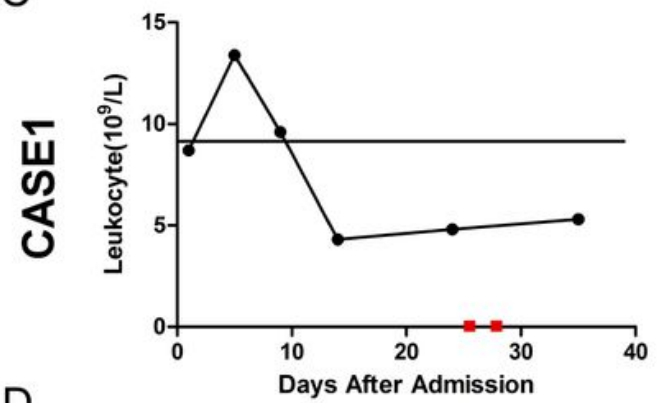

D

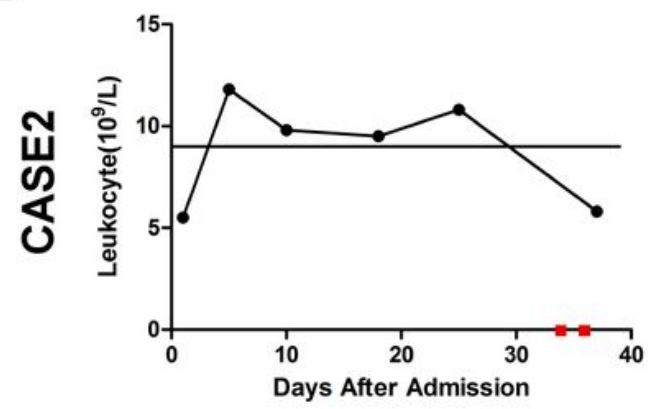

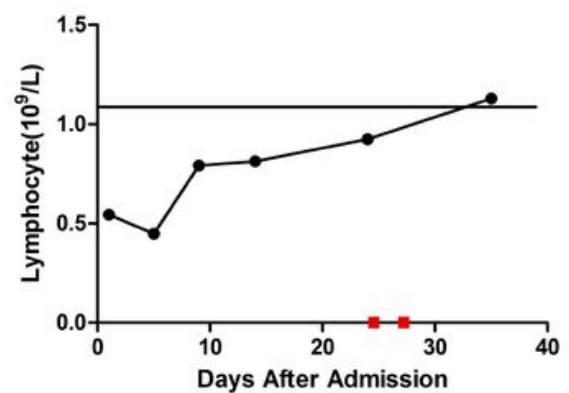

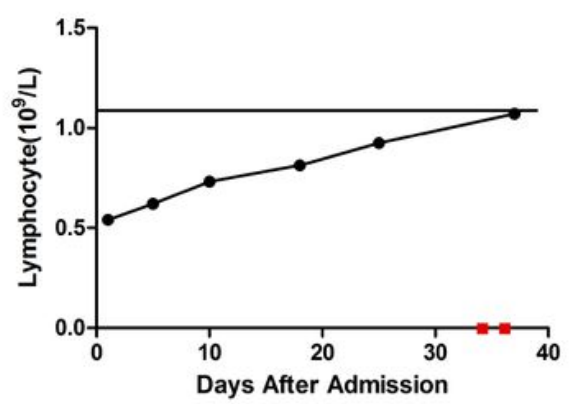

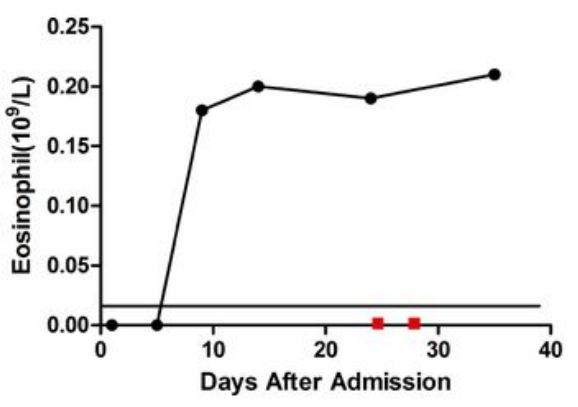

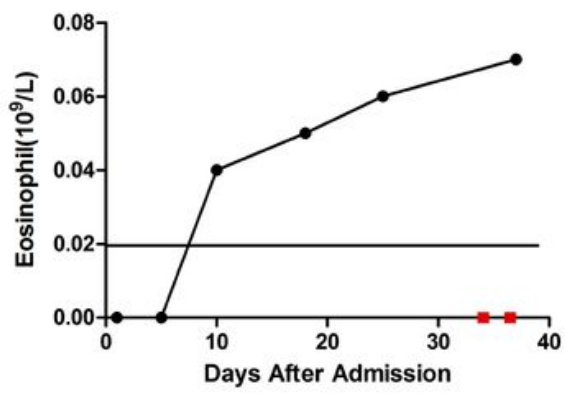

Figure 4

Relationship between blood biomarkers and recovery of patients with COVID-19.A and B. Chest radiographs showing patchy ground-glass opacities (case 1 ) and multifocal opacity changes (case 2 ) overtime. C and D. The temporal changes of laboratory indicators in COVID-19 patients (case 1 and case 
2) after admission. The red dot on the $X$ axis in the figure indicates that the SARS-CoV-2 nucleic acid test result is negative.

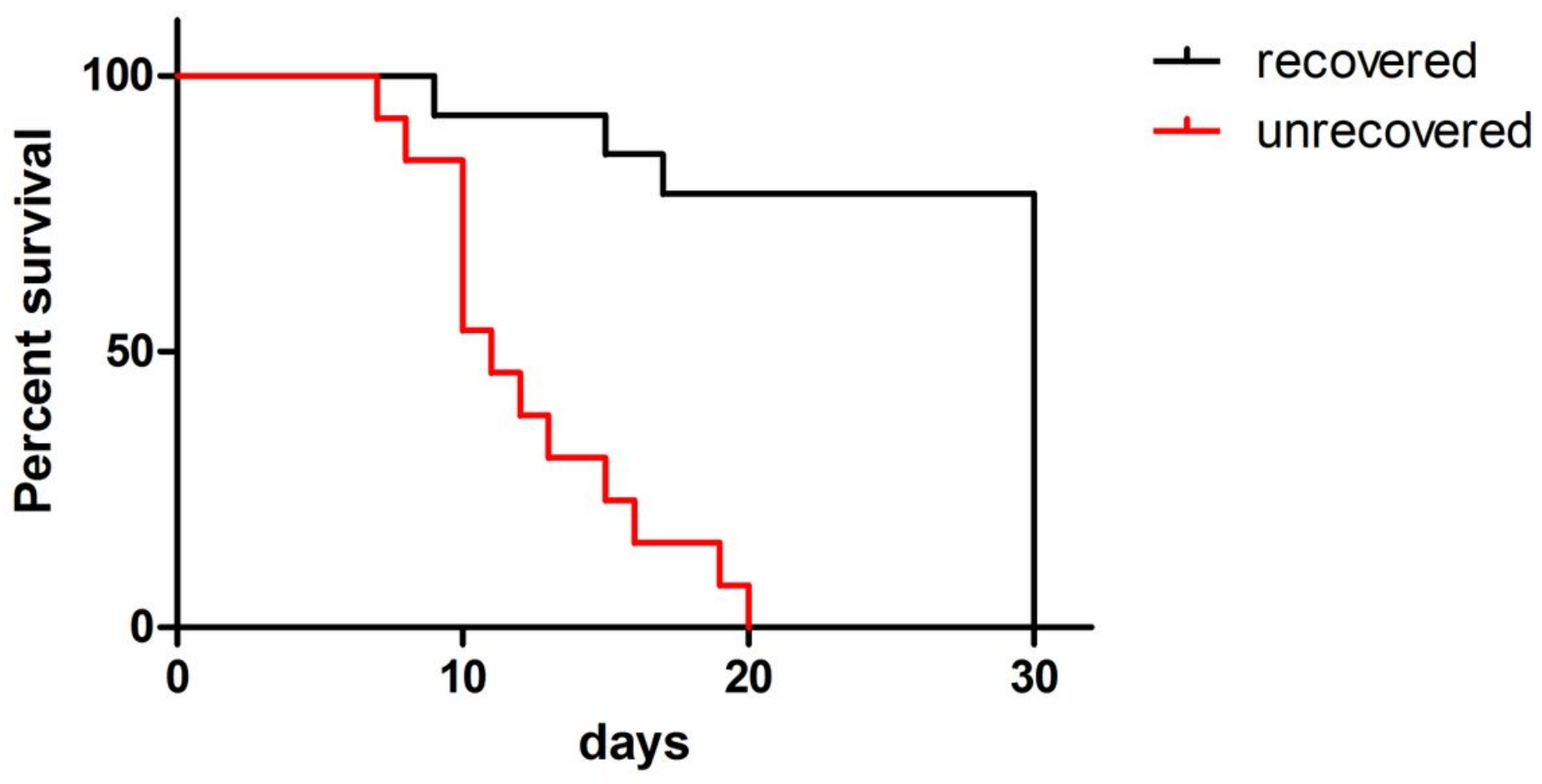

Figure 5

Survival curve related to recovery of patient's eosinophil count. recovered: patients whose eosinophil count returns to normal levels. unrecovered: patients whose eosinophil count have not returned to normal levels. 